\title{
OPTIMAL BEAM PATTERN DESIGN FOR VERY LARGE SENSOR ARRAYS WITH SPARSE SAMPLING
}

\author{
Yenming M. Lai, Radu Balan* \\ University of Maryland \\ AMSC Program and Department of Mathematics \\ College Park, MD 20742
}

\author{
Heiko Claussen, Justinian Rosca
}

\author{
Siemens Corporation \\ Corporate Technology \\ Princeton, NJ 08540
}

Consider a large scale sensor array having $N$ sensors that monitors a surveillance area. Using all sensors simultaneously may be unreasonable in terms of power consumption and data processing. For example, for $N=10000$ sensors and a data sampling rate of 100000 samples per second, the bandwidth requirement is $1 \mathrm{Gsam}$ ples/sec. We could poll a only subset of $D$ sensors at any one given time instead. The $N$ choose $D$ number of choices of sensors allows for a myriad of sensor configurations, and the task is then to choose a subset that achieves our objective.

Assume the surveillance area consists of a set of point-like sources. We seek designs that minimize the largest interference gain from a potentially very large number of locations while still maintaining target unity gain.

Throughout this paper, we assume the following: Sensor locations ( $x, y, z$-coordinates) are known. The number of real interferences is small, but their locations are unknown. The maximum number of sensors active at any time, $D$, is fixed in advance, and all signals are wide band.

Our system divides the surveillance area into a large number of small, non-overlapping target areas. For each fixed target area, we find a subset of sensors that minimizes the gain of a large number of interferences. By doing so, our system is robust to a wide variety of unknown interference configurations. Our system then begins to iteratively scan each target area. By measuring the signal strength in each fixed target area, we are able to locate and hence monitor the target.

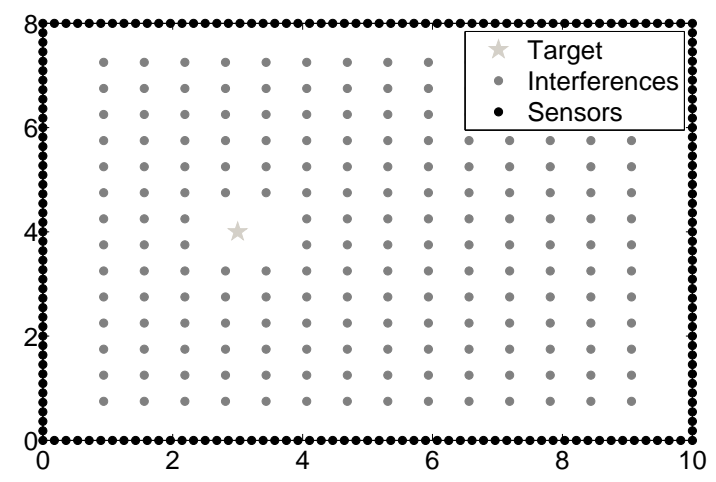

Fig. 1. Sample Scenario: $L=193$ sources ( 1 target and 192 interferences), $N=200$ sensors.

Consider the setup described earlier and sketched in Figure 1. We assume we know the sensor locations and then fix a target area.

${ }^{*}$ Research has been partially supported by NSF under grant DMS1109498, and by Siemens Corporate Research
Since the number, locations, and power distributions of the interferences are unknown, we assume a large number of "virtual" interferences, say $L$. Our objective is to minimize the largest gain from this set of "virtual" interferences which guarantees a small gain for any one actual intereference located at any of the virtual interference locations.

Assume the zero indexed source is the target and the remaining $L$ sources are interferences. For these "virtual" sources we use the direct-path model, where the transfer function between source $l$ and sensors $n$ at frequency $f_{i}$ is given by $H_{l, n}\left(f_{i}\right)$ We use this simple direct-path model because we do not want to assume prior knowledge of wall locations and their reflection coefficients. For evaluation we use the direct-path plus one reflection model.

Let $w_{n}\left(f_{i}\right)$ denote the processing weight for sensor $n$ at frequency $f_{i}$. If $w_{n}\left(f_{i}\right)=0$ then sensor $n$ is inactive at frequency $f_{i}$.

Assume that we have $F$ distinct frequencies of interest, $f_{1}, f_{2}, \ldots, f_{F}$. Given $F$ sets of $N$ sensor weights, each interference $l$ has $F$ different gains, one for each frequency. Note that for each of the $F$ frequencies, we use a different set $N$ sensor weights to calculate the interference gains at that frequency. Our objective is to minimize the maximum sum of gains across $F$ frequencies while still achieving target unit gain at each frequency. Assumption H4, however limits us to $D$ simultaneously active sensors.

In this multi-frequency setup, sensor $n$ becomes active if $w_{n}\left(f_{i}\right) \neq 0$ for any $i$. Thus the number of non-zeros in the $N$ dimensional vector $\max _{1 \leq i \leq F}\left|w_{n}\left(f_{i}\right)\right|$ is then an appropriate measure of the number of active sensors. Let $\|w\|_{0}$ denote the pseudo-norm which counts the number of non-zeros in the vector $w$. We can now state our optimization problem:

$$
\begin{array}{|ll|}
\min _{\left(w_{n}\left(f_{i}\right)\right)_{i=1, n=1}^{F, N}} & \max _{1 \leq l \leq L} \sum_{i=1}^{F}\left|\sum_{n=1}^{N} H_{l n}\left(f_{i}\right) w_{n}\left(f_{i}\right)\right| \\
\text { subject to } & \sum_{n=1}^{N} H_{0 n}\left(f_{i}\right) w_{n}\left(f_{i}\right)=1 \text { for } i=1,2, \ldots, F \\
& \left\|\max _{1 \leq i \leq F}\left|w_{n}\left(f_{i}\right)\right|\right\|_{0} \leq D \\
\hline
\end{array}
$$

Note that this is a non convex optimization problem due to the $l^{0}$ pseudo-norm constraint $\left\|\max _{1 \leq i \leq F}\left|w_{n}\left(f_{i}\right)\right|\right\|_{0} \leq D$.

Our method is inspired by LASSO regularization [1], a regression technique that minimizes the sum of squares of residual errors subject to the $l^{1}$ norm of the coefficients being less than a constant. Similar to the sparse signal and model estimation approach in [2], the 
$l^{0}$ pseudo-norm is replaced by the $l^{1}$ norm $\|w\|_{1}=\sum_{n=1}^{N}\left|w_{n}(f)\right|$ which is then incorporated into the optimization criterion using a Lagrange multiplier $\lambda$. The optimization problem (1) is then replaced by the following convex optimization problem which we call the $\lambda$ method:

$$
\begin{aligned}
& \min _{\left(w_{n}\left(f_{i}\right)\right)} \max _{\substack{F=1 \\
i \leq l \leq L \\
n=1}} \sum_{i=1}^{F}\left|\sum_{n=1}^{N} H_{l n}\left(f_{i}\right) w_{n}\left(f_{i}\right)\right|+\lambda \sum_{n=1}^{N} \max _{1 \leq i \leq F}\left|w_{n}\left(f_{i}\right)\right| \\
& \text { subject to } \sum_{n=1}^{N} H_{0 n}\left(f_{i}\right) w_{n}\left(f_{i}\right)=1 \text { for } i=1,2, \ldots, F
\end{aligned}
$$

For very large $\lambda$, the penalty term forces many of the sensors to become inactive. Specifically, let $W$ be the $L$ by $F$ matrix of sensor weights produced by the optimization. With a large $\lambda$ penalty, many of the rows of $W$ are rows containing only values very close to zero. If the $n$th row only contains such small values, sensor $n$ is then inactive. As $\lambda$ decreases the penalty term becomes less expensive and more sensors become active. At the limit, $\lambda=0$, all sensors are allowed to be active. We fine tune $\lambda$ until we get $D$ nonzero components. Specifically, this is accomplished when $g_{n_{D+1}}$ is significantly smaller than $g_{n_{1}}$ (e.g. by 3 orders of magnitude), where $g_{n}$ is defined as before. We then solve (2) using this lambda. The final weights are obtained by solving a second time (2) restricted to the sub matrix indexed by $\mathcal{A}$ and $\lambda=0$.

Our approach extends the work Ling et al. [3] to include optimization over multiple frequencies simultaenously and reoptimization over the the most significant sensor locations.

We run three types of experiments to test the performance of the $\lambda$-method. First, for small problem sizes (small $N$ ), we do an exhaustive search of all $N$ choose $D$ choices and compare the performance of the set of sensors found by the lambda-method against the performance of the set of sensors which achieves the global optimum. Second, we randomly perturb the the set of sensors found by the $\lambda$-method using the method of simulated annealing to see how much a better of a solution we can find. Third, we compare the performance of the $\lambda$-method beamformer against a beamformer that maximizes the signal to noise ratio (SNR), which can be shown to the set of sensors that lie closet to the target. The below figures show the beam patterns for both the $\lambda$-method and the maximumSNR beamformer at $1000 \mathrm{hz}$ along with the placement of the microphones. We also compare the expected value and variance of the maximum gain among a varying number of randomly placed interferences in the survelliance area. The full paper will include results for the other frequencies $(2000,4000,8000 \mathrm{hz})$ that were simultaneously optimized over.

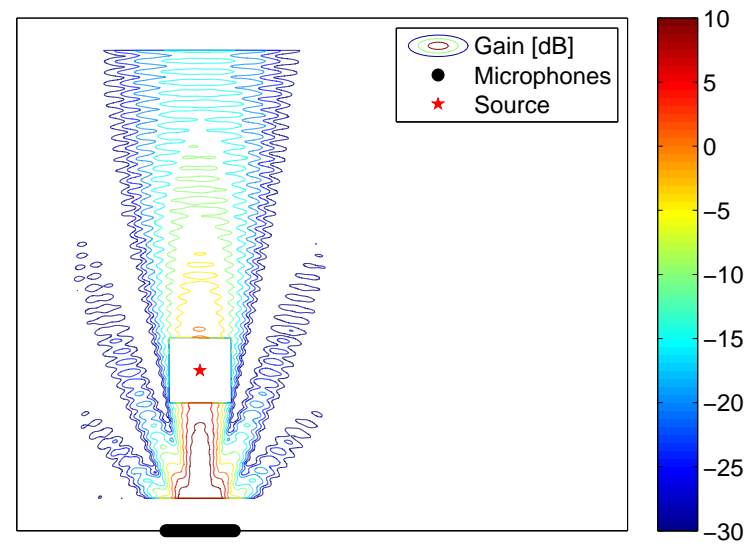

Fig. 2. Gain map for classical beamformer that maximizes SNR

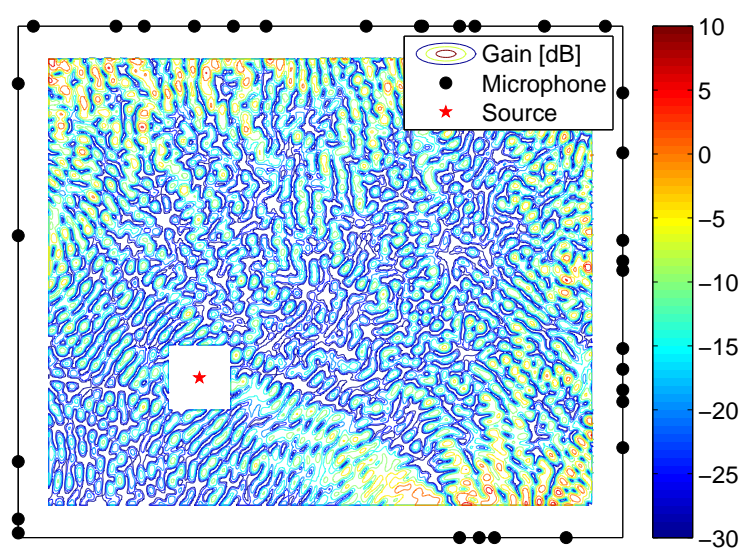

Fig. 3. Gain map for beamformer found by lambda-method

\section{REFERENCES}

[1] R. Tibshirani, "Regression shrinkage and selection via the lasso," Journal of the Royal Statistical Society (Series B), vol. 58, pp. 267-288, 1996.

[2] E.J. Candes and Y. Plan, "Near-ideal model selection by 11 minimization," Annals of Statistics, vol. 37, no. 5A, pp. 2145-2177, 2009.

[3] C. Ling, S. Wee, C. Wei, and Y. Zhu Liang, "Linear sparse array synthesis via convex optimization," in Circuits and Systems (ISCAS), Proceedings of 2010 IEEE International Symposium on, 2010, pp. 4233-4236. 

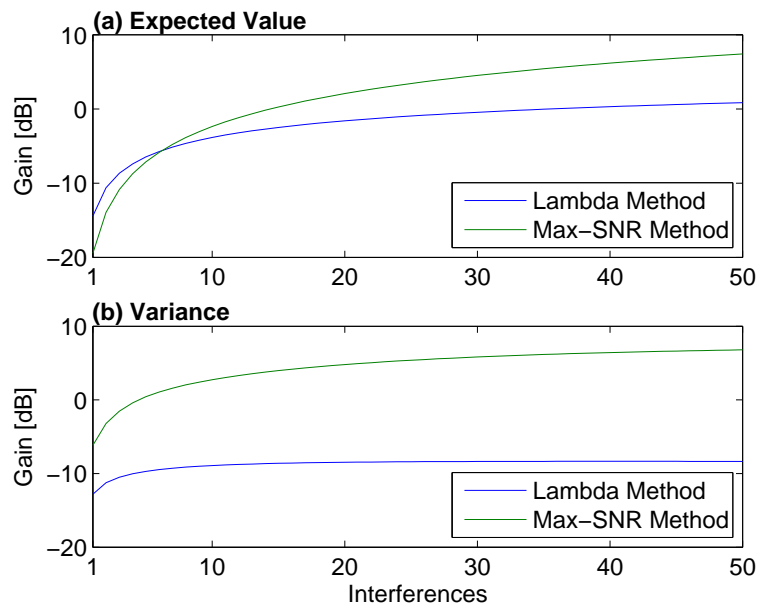

Fig. 4. Comparison of mean and variance of maximum gain for varying number of randomly placed interferences 